\title{
The placental transcriptome of the first- trimester placenta is affected by in vitro fertilization and embryo transfer
}

\author{
Liang Zhao', Xiuli Zheng'1 Jingfang Liu', Rong Zheng ${ }^{1}$, Rui Yang ${ }^{2}$, Ying Wang ${ }^{2}$ and Lifang Sun ${ }^{1 *}$ (D)
}

\begin{abstract}
Background: The placenta is a highly specialized temporary organ that is related to fetal development and pregnancy outcomes, and epidemiological data demonstrate an increased risk of placental abnormality after in vitro fertilization and embryo transfer (IVF-ET).

Methods: This study examines alterations in the transcriptome profile of first-trimester placentas from IVF-ET pregnancies and analyzes the potential mechanisms that play a role in the adverse perinatal outcomes associated with IVF-ET procedures. Four human placental villi from first-trimester samples were obtained through fetal bud aspiration from patients subjected to IVF-ET due to oviductal factors. An additional four control human placental villi were derived from a group of subjects who spontaneously conceived a twin pregnancy. We analyzed their transcriptomes by microarray. Then, RT-qPCR and immunohistochemistry were utilized to analyze several dysregulated genes to validate the microarray results. Biological functions and pathways were analyzed with bioinformatics tools.
\end{abstract}

Results: A total of 3405 differentially regulated genes were identified as significantly dysregulated ( $>2$-fold change; $P<0.05)$ in the IVF-ET placenta in the first trimester: 1910 upregulated and 1495 downregulated genes. Functional enrichment analysis of the differentially regulated genes demonstrated that the genes were involved in more than 50 biological processes and pathways that have been shown to play important roles in the first trimester in vivo. These pathways can be clustered into coagulation cascades, immune response, transmembrane signaling, metabolism, cell cycle, stress control, invasion and vascularization. Nearly the same number of up- and downregulated genes participate in the same biological processes related to placental development and maintenance. Procedures utilized in IVF-ET altered the expression of first-trimester placental genes that are critical to these biological processes and triggered a compensatory mechanism during early implantation in vivo.

Conclusion: These data provide a potential basis for further analysis of the higher frequency of adverse perinatal outcomes following IVF-ET, with the ultimate goal of developing safer IVF-ET protocols.

Keywords: Placenta, In vitro fertilization and embryo transfer (IVF-ET), Gene expression, Human, Adverse perinatal outcomes

\footnotetext{
* Correspondence: lifangsun1966@aliyun.com

${ }^{1}$ Department of Obstetrics and Gynecology, Beijing Jishuitan, Hospital, No.

31, Xinjiekou East Street, Xicheng District, Beijing 100035, People's Republic

of China

Full list of author information is available at the end of the article
}

(c) The Author(s). 2019 Open Access This article is distributed under the terms of the Creative Commons Attribution 4.0 International License (http://creativecommons.org/licenses/by/4.0/), which permits unrestricted use, distribution, and reproduction in any medium, provided you give appropriate credit to the original author(s) and the source, provide a link to the Creative Commons license, and indicate if changes were made. The Creative Commons Public Domain Dedication waiver (http://creativecommons.org/publicdomain/zero/1.0/) applies to the data made available in this article, unless otherwise stated. 


\section{Background}

The widespread application of therapeutic methods for subfertile patients, particularly in vitro fertilization and embryo transfer (IVF-ET), has remarkably increased the pregnancy rate [1]. The elective frozen-thawed singleblastocyst transfer approach, which has been widely adopted in multiple reproductive centers, has largely overcome the risks of multiple pregnancies [2, 3]. Recent census results show that the perinatal outcomes of the most recent groups of patients are better than those of previous groups of patients [4]. This positive trend is due to more appropriate embryo transfer strategies, milder ovarian stimulation, laboratory technological advances, and improved culture media [5]. However, even in singleton pregnancy, after adjustment for maternal confounding factors, the risk of multiple adverse outcomes during the perinatal period, including miscarriage, preterm birth, small for gestational age, low birth weight and gestational hypertension, are higher in IVF-ET pregnancies than in spontaneously conceived pregnancies [6-10].

The placenta, derived from the embryonic trophectoderm, is a highly specialized and adaptive temporary organ and is critical for embryonic development and perinatal outcomes [11]. Some animal experiments have shown that placental tissue is more sensitive to external disturbances than embryonic tissue [12, 13]. The possible explanation for this result is twofold. On the one hand, the trophectoderm, which subsequently develops into the placenta, is the first differentiated cell lineage of the embryo to address various changes in the external environment [14]. On the other hand, the trophectoderm, whose genomic imprints are unstable and vulnerable to the surrounding microenvironment during implantation, appears to be sensitive to in vitro culture conditions and prone to an imbalance in imprinted gene expression $[15,16]$. Although the composition of the culture medium has continuously been improved, it is still not completely equivalent to the physiological conditions of the natural environment in vivo [17]. In addition, even with careful and gentle manipulation, the trophectoderm must withstand severe stress and interference from the environment, such as changes in temperature, $\mathrm{pH}$, and oxygen tension; light exposure; $\mathrm{pH}$ variations during manipulation; and shear stress linked to repeated pipetting, which may interact and synergistically affect placental function and development [18-20].

Professionally, there is an urgent need to understand and determine the impact of IVF-ET on placental development as soon as possible. Although several welldesigned studies, which are limited in terms of research design and material acquisition, have focused on the effects of IVF-ET on human placental function and development, these studies used term placentas. These data support the hypothesis that altered global gene expression leads to overrepresented biological pathways in the human term placenta after IVF-ET [21, 22]. Similarly, term and late-gestation placentas have been examined in animal models, and genome-wide mRNA expression revealed that IVF-ET significantly affects placental functions and triggers compensatory responses [23-25]. Due to alterations in the peri-implantation environment and to key functions in placental development, such as cell fusion, embryo implantation, immune tolerance, and tissue remodeling, investigating the influence of IVF-ET on first-trimester placental development and function is particularly important [26, 27]. In addition, according to maternal gestational physiology and the theory of fetal origins of adult disease [28, 29], related research is needed to determine IVF-ET effects on placental gene expression and biological function as soon as possible, which would contribute toward developing strategies to prevent these diseases.

In this study, we hypothesized that altered global gene expression occurs in placental tissue during the first trimester after IVF-ET compared with the first trimester after spontaneous conception. Specifically, we used a twin-to-singleton selective fetal reduction strategy to obtain first-trimester placentas in vivo. Our previous study using immunohistochemistry showed that IVF-ET affects first-trimester placental development and function [30]. Therefore, we carried out a microarray analysis to investigate the effects of IVF-ET treatment on gene expression and potential biological functions in human firsttrimester placentas compared with human first-trimester placentas of spontaneous pregnancies. Furthermore, a better understanding of the placental mechanisms triggered by IVF-ET per se may be of future value in improving the safety of IVF-ET protocols.

\section{Methods \\ Study subjects}

From January to October 2018, twin-to-singleton selective fetal reduction was performed in 4 cases in the first trimester after IVF-ET. All patients from fresh embryo transfers underwent standard IVF-ET due to oviductal obstruction. The sperm quality test results of each male were normal. In this study, the reason for fetal reduction from twin to singleton was patient preference. The application of the IVF-ET clinical technology was licensed and authorized by the National Health Commission of the People's Republic of China. The control group included 4 cases of unwanted spontaneously conceived monochorionic twins in the same period. Clinical data were collected at the obstetrics outpatient clinic at Beijing Jishuitan Hospital and organized in a database. The number of specimens needed for the analysis of 
variance in the microarray experiment, which was 4 , was determined by the study's statistical criteria. Information about the couple's weights, smoking habits, alcohol consumption habits and hormone use was collected by questionnaire. Information on maternal age, gestational weeks, gravidity history and body mass index (BMI) was matched between the IVF-ET and spontaneously conceived groups.

\section{Sample collection and ethics}

For all cases in which a singleton was retained from a twin pregnancy, reductions were performed by the same senior physician by fetal bud aspiration under ultrasound guidance. We selected 4 cases for villi suction simultaneously. We used a special needle that was authorized by the State Intellectual Property Office of People's Republic of China (No ZL 2015 20,035,543.7). The placental tissue was collected 30-35 days after embryo transfer, which is equivalent to 7-8 gestational weeks. The pregnancies in the control group of double chorionic twin pregnancies were diagnosed with early intrauterine pregnancy based on urine pregnancy tests and transvaginal ultrasound assessments. None of the patients had taken steroid hormone drugs for the previous 3 months and had a record of regular menstruation. The villi were collected during the conventional artificial abortion operation. The villi samples were isolated and purified immediately from the specimens according to the morphology within 30 min under an inverted microscope. All placental tissues were refloated with ice-cold PBS and then stored under RNase-free conditions at $80^{\circ} \mathrm{C}$ until future tissue homogenization and total RNA extraction. Another portion of the remaining specimens was transferred to $4 \%$ paraformaldehyde within $30 \mathrm{~min}$ after operation and incubated at $4{ }^{\circ} \mathrm{C}$ overnight. Then, the specimens were stored in $1 \%$ paraformaldehyde at $4{ }^{\circ} \mathrm{C}$ until routine dehydration and embedding in paraffin. All study participants were enrolled at the Beijing Jishuitan Hospital 2018, were of Asian ancestry and were living in Beijing.

\section{RNA extraction}

Tissue homogenization and total RNA extraction were accomplished at CapitalBio Corporation, Bejing, China, using the Macherey-Nagel NucleoSpin RNA II kit (Macherey-Nagel, Duren, Germany), where a significant reduction in genomic DNA and protein contamination was achieved. Total RNA was derived from approximately $200 \mathrm{mg}$ of first-trimester placental tissue, including various placental types. A NanoDrop ND100 spectrophotometer (NanoDrop Technologies, USA) was used to evaluate the purity and concentration of isolated total RNAs. The intensity ratio of ribosomal $28 \mathrm{~S}$ and
18S RNA was 1.5-1.8:1. The RNA was used in both the microarray assay and subsequent RT-qPCR analysis.

\section{Microarray hybridization}

Thirty nanograms of total RNA was amplified and transcribed into biotinylated cRNA according to the instructions provided in the MessageAmp II aRNA Amplification Kit (Ambion Inc., Carlsbad, Calif., USA). Samples of cDNA were hybridized to the Affymetrix $\mathrm{Hu}$ man Genome U133 Plus 2.0 Array. The cDNA of each sample was hybridized to one Array. This array contains approximately 54,000 oligonucleotide probes and covers more than 30,000 human genes mapped through UniGene or via RefSeq annotation (Affymetrix, Santa Clara, CA, USA). Then, the arrays were washed and stained on the Fluidics Station 450 (Affymetrix) and scanned on an Affymetrix GeneChip Scanner 3000 (Affymetrix) to analyze the hybridization data according to the manufacturer's instructions. The data were collected from the scanned images and evaluated using the Affymetrix GeneChip Operating Software (GCOS 1.4). Differentially expressed genes between the IVF-ET and control groups were analyzed using Significance Analysis of Microarrays (SAM version 3.02, Stanford University, Stanford, CA).

\section{Microarray data analysis}

Our primary microarray data for the first-trimester placental samples have been submitted to the Gene Expression Omnibus of the National Center for Biotechnology Information [31]. The data can be obtained through GEO Series accession number GSE 122214 (https:// www.ncbi.nlm.nih.gov/geo/query/acc.cgi?acc=

GSE122214). Data quality, linear modeling and significance analysis were performed by means of Bioconductor package linear models [32]. The cut-off value was determined to be a twofold difference in expression values. The proportion of false-positive corrections of $<$ 0.05 was considered statistically significant. If a gene had multiple probe identification data in the results, the average fold change was calculated.

To assess the variability in placental tissues with nonparametric methods, hierarchical clustering and principal component analysis were performed to visualize global variation. Based on the difference in placental gene expression between IVF-ET pregnancy and spontaneously conceived pregnancy, a three-dimensional scatter plot was used to visualize the sample gene variation in the principal component analysis. The distance between different points was calculated using the number and covariance of differentially expressed genes. The complete-linkage hierarchical clustering algorithm was used to assess the variation among gene expression profiles. 
Enrichment and integrated analysis of differentially regulated genes were performed by submitting data to Database for Annotation, Visualization and Integrated Discovery (DAVID), Ingenuity Pathway Analysis (IPA), Kyoko Encyclopedia of Genes and Genomes (KEGG), and KEGG Orthology Based Annotation System (KOBAS). According to Gene Ontology terms, DAVID divided blocks of functionally related differentially expressed genes based on biological process, molecular function and cellular component. The other three databases were used to visually integrate dysregulated genes and identify statistically significant enriched pathways. Up- and downregulated genes were investigated separately. Statistical controls were based on all human genes included in the Affymetrix Human Genome U133 Plus 2.0 Array. A false discovery rate $(\mathrm{FDR})<0.05$ and $p$ value $<0.05$ were used to determine statistical significance.

\section{Quantitative real-time RT-PCR}

Validation of the microarray results was performed by real-time RT-qPCR using the same samples for microarray. Synthesis of cDNA was achieved using the PrimeScript RT Reagent Kit (TaKaRa Biotechnology, Dalian, China) with $2 \mu \mathrm{g}$ of RNA. RT-qPCR was performed with an ABI Prism 7300 Sequence Detection System (Applied Biosystems, Roche, USA) according to recommendations of the manufacturer. The following genes were used for microarray validation: alpha-fetoprotein (AFP); glial cells missing homolog 1 (Drosophila) (GCM1); leukocyteassociated immunoglobulin-like receptor 2 (LAIR2); phosphatase and tension homolog (PTEN); B-cell CLL/ lymphoma 2 (BCL2); transferrin (TF); metallothionein 1 M (MT1M); epidermal growth factor receptor (EGFR); tubulin, beta 1 class VI (TUBB1); and vascular endothelial growth factor (VEGF). Considering their critical functions in the first-trimester placenta based on the biological analysis, these ten target genes, which showed differential expression in the microarray analysis, were selected and tested by RT-qPCR. Three technical and five biological replicates were run for all RT-qPCR analyses. Relative mRNA expression levels were calculated using the comparative threshold $\Delta \Delta \mathrm{Ct}$ method. Comparison of gene expression between the IVF-ET and control groups was performed using a two-tailed Student's $t$-test to determine significant differences. $P<0.05$ was considered significant. A list of all predesigned PCR primer pairs used in the RT-qPCR analysis is provided (Additional file 1: Table S1).

\section{Immunohistochemistry (IHC)}

The first-trimester placental tissues used for immunohistochemical staining originated from the same tissues used for the previous RNA extractions. The placental tissues that were stored in PFA/PBS were fixed and embedded in paraffin. Then, these samples were deparaffinized, rehydrated and sectioned. Immunohistochemical localization was performed to analyze the AFP, VEGF, TF, TUBB1, BCL2, GCM1, PTEN and LAIR2 gene products using $5-\mu \mathrm{m}$ paraffin sections mounted on Superfrost Plus slides (Gerhard Menzel, Braunschweig, Germany). The primary antibodies utilized were as follows: AFP (1:25 dilution, ZM-0009; ZSGB-Bio, Beijing, China), VEGF (1:25 dilution, ZA0509, ZSGB-Bio, Beijing, China), TF (1:25 dilution, TA500848, ZSGB-Bio, Beijing, China), TUBB1 (1:25 dilution, TA506588, ZSGBBio, Beijing, China), BCL2 (1:25 dilution, ZA-0010; ZSGB-Bio, Beijing, China), GCM1 (1:100 dilution, ab187860; Abcam, Cambridge, UK), PTEN ((1:25 dilution, ZA-0635; ZSGB-Bio, Beijing, China), and LAIR2 (1: 100 dilution, ab183145, Abcam, Cambridge, UK). Protein detection and negative control assessment were conducted simultaneously following the manufacturer's instructions. A microscope magnification of $\times 400$ was used. Two senior pathologists examined the stained sections in a double-blinded fashion and performed the quantifications manually. The positivity rates are described as stained cells vs the total number of cells.

\section{Results}

\section{Characteristics of the patients}

In terms of age, gestational age, parity and BMI, there were no significant differences between the women who were subjected to IVF-ET and those who spontaneously conceived (Table 1).

\section{Microarray data}

Transcriptomic profiling of 8 human first-trimester placental samples was performed on samples obtained from 4 IVF-ET and 4 spontaneously conceived pregnancies. A total of 3405 differentially expressed genes were validated according to a false discovery rate of 0.05 . A total of 1910 genes were expressed at higher levels, and 1495 genes were expressed at lower levels in the firsttrimester placentas produced by IVF-ET compared with those produced by natural conception. Interestingly, the numbers of upregulated (56.1\%) and downregulated (43.9\%) genes were well balanced (Fig. 1a, b, d).

Hierarchical clustering analysis of the microarray data demonstrated an expressional alteration and separation of placental dysregulated transcripts between the IVFET and control groups (Fig. 1a). Alternatively, the principal component analysis was applied to placental gene expression profiles and demonstrated the same separate pattern, where the two study groups clustered into two separate groups (Fig. 1c).

The functional enrichment annotation of differentially expressed genes demonstrated that more than 50 Gene 
Table 1 Characteristics of the patients in the two groups

\begin{tabular}{llllll}
\hline & $N$ & Mean age & Gestational age (day) & Parity & BMl (kg/m2) \\
\hline IVF-ET group & 4 & $30.66 \pm 3.76$ & $49.44 \pm 3.14$ & $1.10 \pm 1.21$ & $23.33 \pm 3.17$ \\
Control group & 4 & $28.86 \pm 3.45$ & $49.35 \pm 3.23$ & $2.21 \pm 0.89$ & $22.99 \pm 2.35$ \\
$\mathrm{t}$ & & 0.833 & 0.637 & 0.858 & 1.311 \\
$\mathrm{p}$ & & 0.425 & 0.529 & 0.437 & 0.263 \\
\hline
\end{tabular}




Ontology (GO) categories and pathways were successfully clustered in the list of dysregulated transcripts. Many broad biological processes of upregulated genes in pregnancy progression were regulation of transcription DNA-dependent $\left(n=149\right.$, FDR $\left.P=1.15 \times 10^{-124}\right)$, transcription $\left(n=143\right.$, FDR $\left.P=1.55 \times 10^{-111}\right)$, oxidation reduction $\left(n=82\right.$, FDR $\left.P=7.98 \times 10^{-96}\right)$, development $(n=84$, FDR $\left.P=1.95 \times 10^{-49}\right)$, and cell adhesion $(n=50$, FDR $P=7.37 \times$ $10^{-43}$ ). The biological processes of the downregulated genes were regulation of transcription DNA-dependent $(n=102$ FDR $\left.P=8.38 \times 10^{-75}\right)$, signal transduction $(n=103$, FDR $\left.P=4.54 \times 10^{-58}\right)$, transcription $(n=87$, FDR $P=5.65 \times$ $\left.10^{-55}\right)$, proteolysis $\left(n=53\right.$, FDR $\left.P=8.86 \times 10^{-43}\right)$, and development $\left(n=72\right.$, FDR $\left.P=6.00 \times 10^{-42}\right)$. Additional biological processes for the up- and downregulated genes are listed (Additional file 2: Table S2 Additional file 3: Table S3). Interestingly, almost the same numbers of up- and downregulated genes are allocated in the same biological process related to placental development and maintenance (Fig. 2). These differentially expressed genes were widely distributed to the nucleus, cytoplasm and cell membrane of the placenta. The GO analyses also highlight the disturbances in various gene and molecular functions and their link to pregnancy complications.
In the IPA, some networks were built based on the biological processes of differentially regulated placental gene expression in the IVF-ET group (Table 2). These abnormal pathways included metabolic pathways $(n=$ 279, FDR $P=7.43 \times 10^{-22}$ ), complement and coagulation cascades $\left(n=44\right.$, FDR $\left.P=5.74 \times 10^{-14}\right)$, Proteoglycans in cancer $\left(n=58\right.$, FDR $\left.P=4.17 \times 10^{-8}\right)$, the PPAR signaling pathway $\left(n=23\right.$, FDR $\left.P=1.08 \times 10^{-4}\right)$, the Wnt signaling pathway $\left(n=35\right.$, FDR $\left.P=1.53 \times 10^{-4}\right)$, and the Jak-STAT signaling pathway $\left(n=36\right.$, FDR $\left.P=5.50 \times 10^{-4}\right)$, among others. For significant proteoglycans in cancer and the PPAR signaling pathway, we labeled the up- (red) and downregulated (green) genes in the signaling pathways (Fig. 3, Additional file 4: Figure S1).

\section{Quantitative RT-PCR}

RT-qPCR analysis of ten selected genes was performed to validate the microarray data. These ten differentially regulated genes were selected according to their biological processes, which were mostly related to placental functions (Fig. 4). The RT-qPCR results confirmed the upregulation the AFP, VEGF, and TF genes and the downregulation of the BCL2, GCM1, EGFR, PTEN, LAIR2, TUBB1, and MT1G genes in the first-trimester

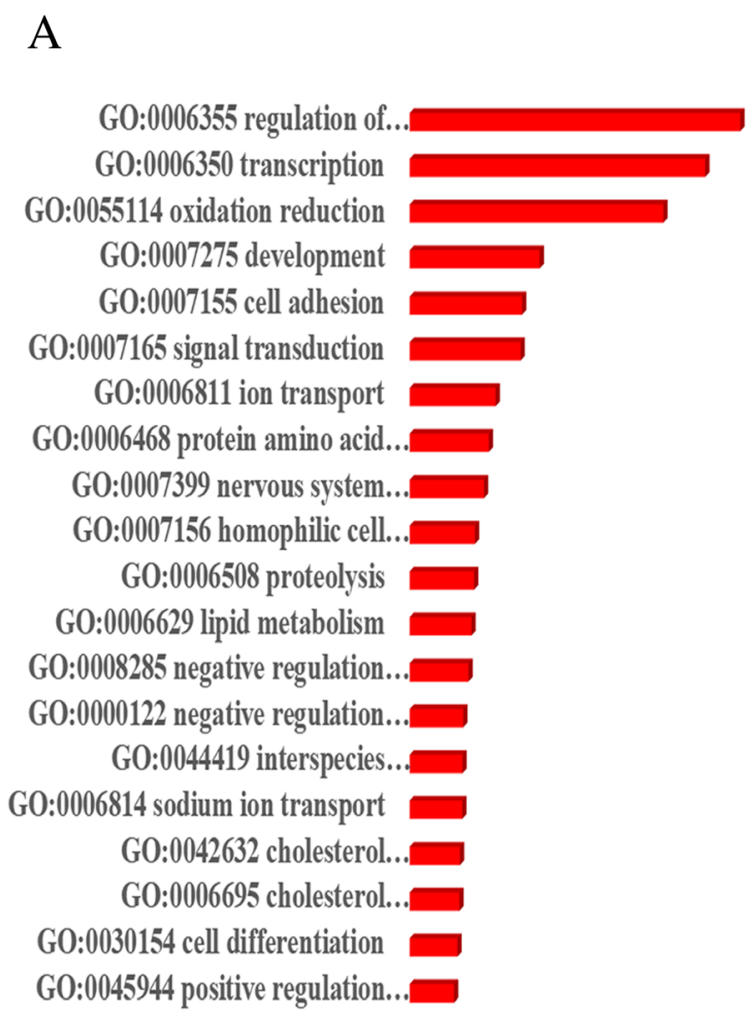

$\begin{array}{llllllll}0 & 20 & 40 & 60 & 80 & 100 & 120 & 140\end{array}$

\section{B}

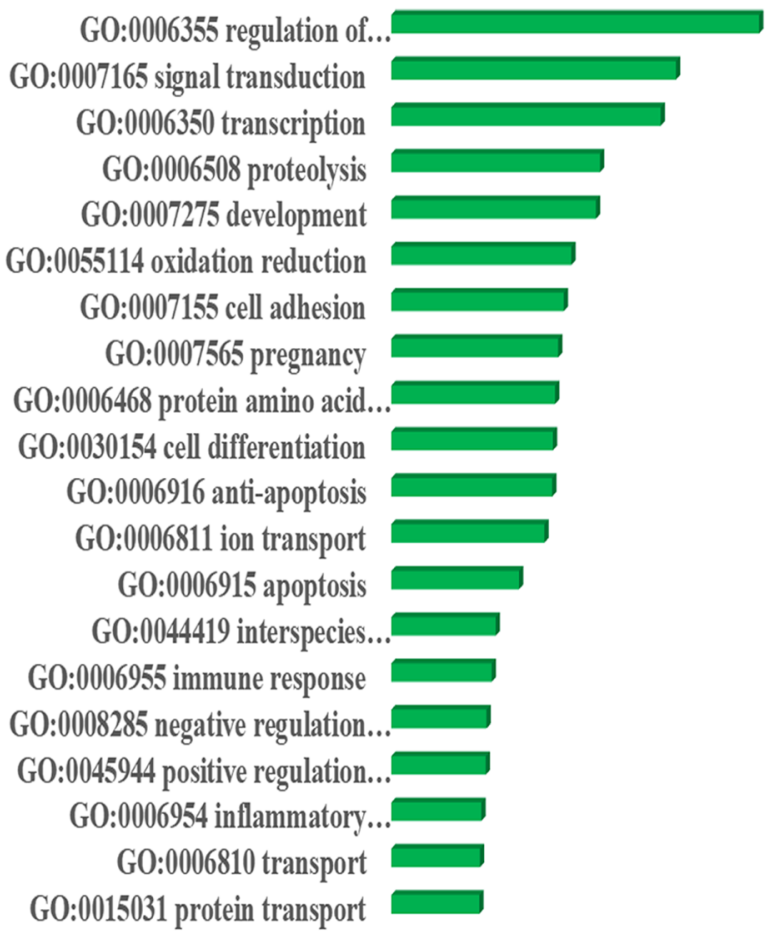

$\begin{array}{lllllllll}0 & 10 & 20 & 30 & 40 & 50 & 60 & 70 & 80\end{array}$

Fig. 2 Functional enrichment analysis of differentially regulated genes in the placenta after IVF-ET compared with the control group during the first trimester according to biological processes. Red, upregulated genes; green, downregulated genes 
Table 2 Cluster of significant pathways related to IVF-ET

\begin{tabular}{|c|c|c|c|c|}
\hline No & Term & ID & Input number & $P$-Value \\
\hline 1 & Metabolic pathways & hsa01100 & 279 & $7.43 \times 10^{-22}$ \\
\hline 2 & Complement and coagulation cascades & hsa04610 & 44 & $5.74 \times 10^{-14}$ \\
\hline 3 & Proteoglycans in cancer & hsa05205 & 58 & $4.17 \times 10^{-8}$ \\
\hline 4 & Pathways in cancer & hsa05200 & 87 & $3.72 \times 10^{-7}$ \\
\hline 5 & Insulin resistance & hsa04931 & 33 & $1.08 \times 10^{-5}$ \\
\hline 6 & Carbon metabolism & hsa01200 & 33 & $1.46 \times 10^{-5}$ \\
\hline 7 & Axon guidance & hsa04360 & 44 & $2.23 \times 10^{-5}$ \\
\hline 8 & Ras signaling pathway & hsa04014 & 52 & $3.77 \times 10^{-5}$ \\
\hline 9 & Glycine, serine and threonine metabolism & hsa00260 & 17 & $4.59 \times 10^{-5}$ \\
\hline 10 & Mineral absorption & hsa04978 & 19 & $8.74 \times 10^{-5}$ \\
\hline 11 & PPAR signaling pathway & hsa03320 & 23 & $1.08 \times 10^{-4}$ \\
\hline 12 & Wnt signaling pathway & hsa04310 & 35 & $1.53 \times 10^{-4}$ \\
\hline 13 & Transcriptional misregulation in cancer & hsa05202 & 41 & $1.72 \times 10^{-4}$ \\
\hline 14 & AGE-RAGE signaling pathway in diabetic complications & hsa04933 & 28 & $1.74 \times 10^{-4}$ \\
\hline 15 & HIF-1 signaling pathway & hsa04066 & 28 & $2.28 \times 10^{-4}$ \\
\hline 16 & mTOR signaling pathway & hsa04150 & 36 & $3.31 \times 10^{-4}$ \\
\hline 17 & Fatty acid metabolism & hsa01212 & 17 & $3.32 \times 10^{-4}$ \\
\hline 18 & Focal adhesion & hsa04510 & 44 & $3.81 \times 10^{-4}$ \\
\hline 19 & Regulation of actin cytoskeleton & hsa04810 & 46 & $3.91 \times 10^{-4}$ \\
\hline 20 & Fatty acid degradation & hsa00071 & 16 & $3.94 \times 10^{-4}$ \\
\hline 21 & Steroid hormone biosynthesis & hsa00140 & 19 & $3.94 \times 10^{-4}$ \\
\hline 22 & Bile secretion & hsa04976 & 21 & $4.29 \times 10^{-4}$ \\
\hline 23 & Aldosterone-regulated sodium reabsorption & hsa04960 & 15 & $4.65 \times 10^{-4}$ \\
\hline 24 & Jak-STAT signaling pathway & hsa04630 & 36 & $5.50 \times 10^{-4}$ \\
\hline 25 & ErbB signaling pathway & hsa04012 & 24 & $6.18 \times 10^{-4}$ \\
\hline 26 & Steroid biosynthesis & hsa00100 & 10 & $6.33 \times 10^{-4}$ \\
\hline 27 & Valine, leucine and isoleucine degradation & hsa00280 & 16 & $7.02 \times 10^{-4}$ \\
\hline 28 & Renal cell carcinoma & hsa05211 & 20 & $7.19 \times 10^{-4}$ \\
\hline 29 & Biosynthesis of amino acids & hsa01230 & 21 & $9.01 \times 10^{-4}$ \\
\hline 30 & Tyrosine metabolism & hsa00350 & 13 & $9.74 \times 10^{-4}$ \\
\hline 31 & Tryptophan metabolism & hsa00380 & 14 & $1.01 \times 10^{-3}$ \\
\hline 32 & EGFR tyrosine kinase inhibitor resistance & hsa01521 & 22 & $1.09 \times 10^{-3}$ \\
\hline 33 & Cytokine-cytokine receptor interaction & hsa04060 & 51 & $1.10 \times 10^{-3}$ \\
\hline 34 & Arginine biosynthesis & hsa00220 & 10 & $1.11 \times 10^{-3}$ \\
\hline 35 & Proximal tubule bicarbonate reclamation & hsa04964 & 10 & $1.45 \times 10^{-3}$ \\
\hline 36 & Sphingolipid metabolism & hsa00600 & 15 & $1.46 \times 10^{-3}$ \\
\hline 37 & Rap1 signaling pathway & hsa04015 & 43 & $1.48 \times 10^{-3}$ \\
\hline 38 & Hippo signaling pathway-multiple species & hsa04392 & 11 & $1.64 \times 10^{-3}$ \\
\hline 39 & FoxO signaling pathway & hsa04068 & 30 & $1.79 \times 10^{-3}$ \\
\hline 40 & Glycerolipid metabolism & hsa00561 & 17 & $1.84 \times 10^{-3}$ \\
\hline 41 & Vitamin digestion and absorption & hsa04977 & 10 & $1.87 \times 10^{-3}$ \\
\hline 42 & Pentose phosphate pathway & hsa00030 & 11 & $2.05 \times 10^{-3}$ \\
\hline 43 & CAMP signaling pathway & hsa04024 & 40 & $2.13 \times 10^{-3}$ \\
\hline 44 & AMPK signaling pathway & hsa04152 & 28 & $2.26 \times 10^{-3}$ \\
\hline
\end{tabular}


Table 2 Cluster of significant pathways related to IVF-ET (Continued)

\begin{tabular}{lllll}
\hline No & Term & ID & Input number & $P$-Value \\
\hline 45 & Arginine and proline metabolism & hsa00330 & 15 & $2.41 \times 10^{-3}$ \\
46 & Alanine, aspartate and glutamate metabolism & hsa00250 & 12 & $2.60 \times 10^{-3}$ \\
47 & Epithelial cell signaling in Helicobacter pylori infection & hsa05120 & 18 & $3.00 \times 10^{-3}$ \\
48 & beta-Alanine metabolism & hsa00410 & 11 & $3.13 \times 10^{-3}$ \\
49 & Insulin signaling pathway & hsa04910 & 30 & $3.13 \times 10^{-3}$ \\
50 & Propanoate metabolism & hsa00640 & 11 & $3.81 \times 10^{-3}$ \\
\hline
\end{tabular}

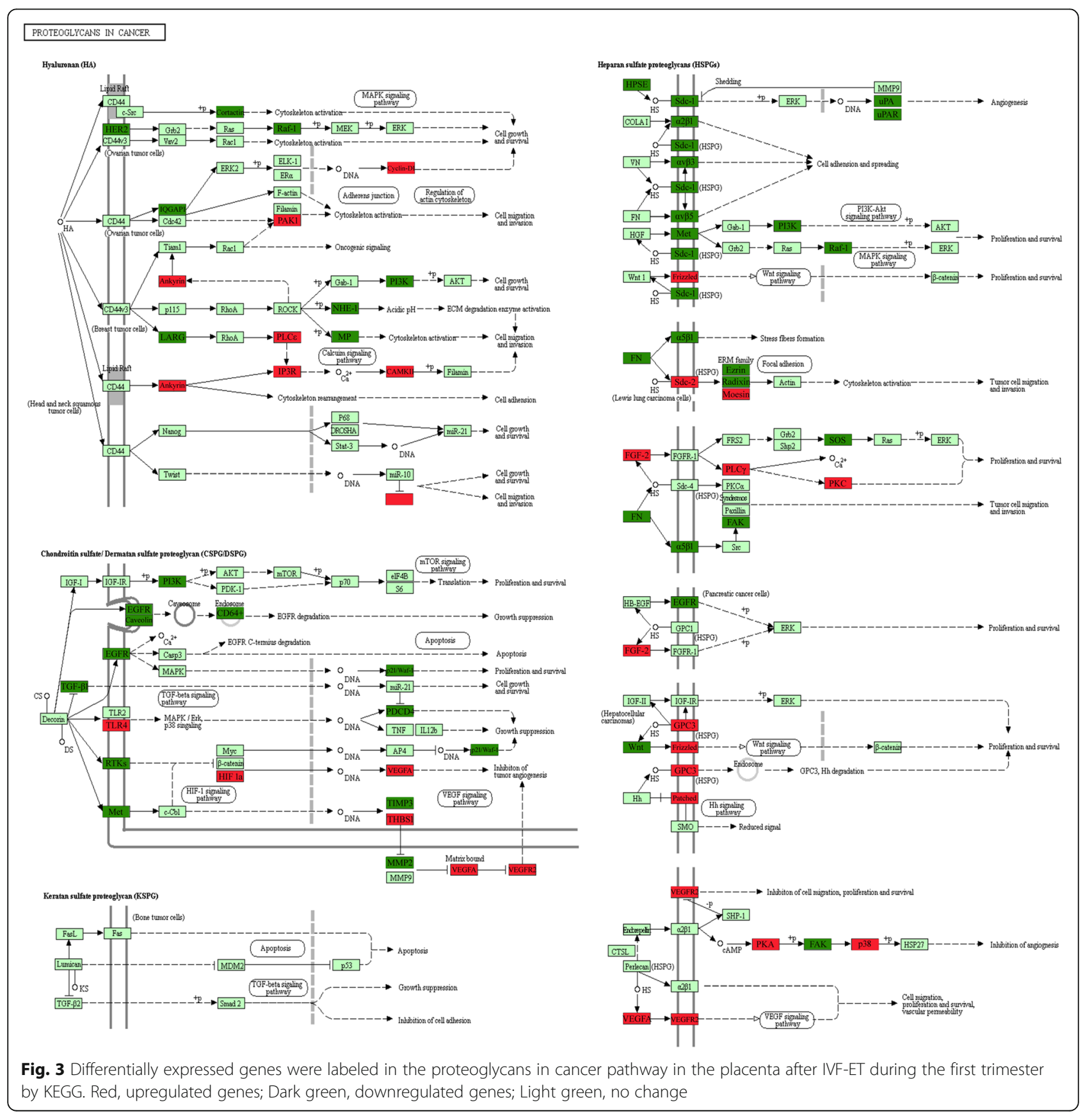



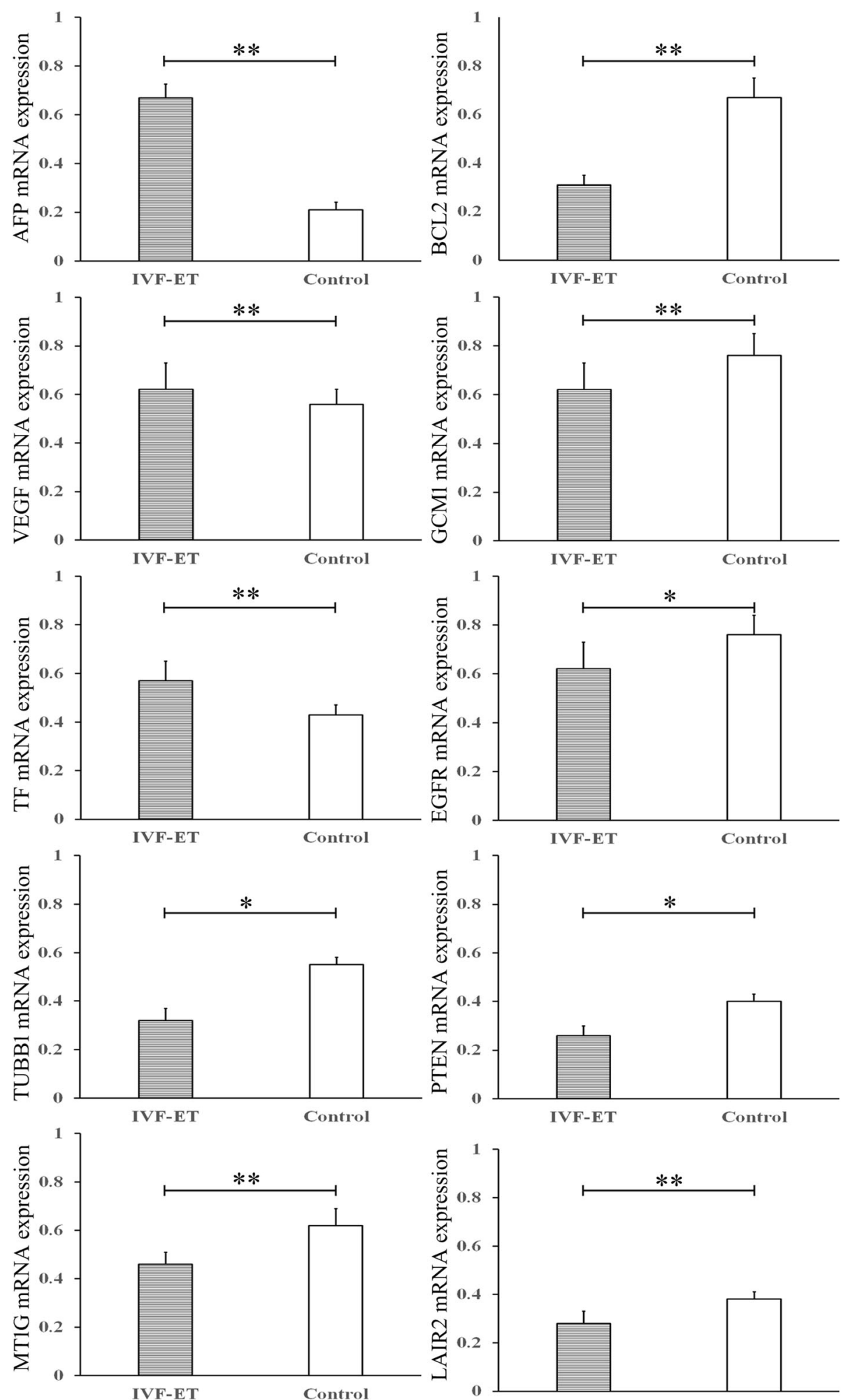

Fig. 4 Validation by RT-qPCR for selected genes from first-trimester placentas derived from IVF-ET compared with those derived from the control group. The mRNA levels of AFP, GCM1, LAIR2, PTEN, BCL2, TF, MT1M, EGFR, VEGF, and TUBB1 in the IVF-ET and control groups were examined by RT-qPCR. All data are expressed as the mean \pm standard deviation, ${ }^{*}$ indicates $P<0.05$; ** indicates $P<0.01$ 
placenta after IVF-ET compared with that after natural conception. We observed a significant concordance among the ten tested genes with the array hybridization obtained previously and confirmed more accurate gene expression differences.

\section{Immunohistochemistry}

The localizations of the AFP, VEGF, TF, TUBB1, BCL2, GCM1, PTEN and LAIR2 proteins, which represent different biological processes in placentation, were investigated in the human first-trimester placenta (Fig. 5). These 8 genes were mainly localized to the cytoplasm, cell membrane and intercellular spaces of the human first-trimester placenta. In addition, both the IVF-ET group and control group showed expression of these 8 genes. A larger number of cells showed strong AFP, VEGF and TF staining, with cytoplasmic staining, in the first-trimester IVF-ET placental group than in the control group. The control group had higher expression of TUBB1, BCL2, GCM1, PTEN and LAIR2 than the IVFET group. The immunohistochemical expression of these genes is consistent with the results of the microarray and RT-qPCR.

\section{Discussion}

Epidemiological studies have demonstrated that even singleton pregnancies conceived after IVF-ET are more likely to develop prematurity, gestational hypertension, placental abruption, low birth weight and intrauterine growth retardation [6-10]. The risk of adverse outcomes originating from suboptimal placentation in pregnancies after IVF-ET is significantly greater than that in spontaneously conceived pregnancies [33, 34]. Several metaanalysis and animal-model studies have demonstrated that this phenomenon is due not only to the infertility diagnosis but also to IVF-ET itself [35-37]. Early placental implantation involves a series of important events, namely, early trophoblast migration, cell fusion, tissue remodeling, immune tolerance, metabolism and extracellular matrix degradation [38-40]. As these trophoblast properties disappear at the end of pregnancy [41, 42], it is particularly critical to study the effects of IVFET on first-trimester placental gene expression. Here, we demonstrated that IVF-ET pregnancies show altered expression of first-trimester placental genes that are critical to development and function compared with spontaneously conceived pregnancies. Genome-wide mRNA expression analysis of the first-trimester placenta after IVF-ET also revealed 50 significantly overrepresented biological pathways. Our findings provide evidence of functional placental pathology and a potential basis for understanding the increased risk associated with the first-trimester placenta following IVF-ET.
In the present study, we identified and confirmed that there was a significant difference in first-trimester placental gene expression between IVF-ET and spontaneous pregnancies. We report 3405 differentially expressed genes, including 1910 upregulated and 1495 downregulated genes. These genes were roughly clustered according to their biological functions: cellular process, physiological, biological regulation, metabolism, regulation of biological process, multicellular organismal process, catalytic activity, developmental process, binding and other functions. Our results are consistent with those of most recent studies and support the hypothesis that IVF-ET procedures affect placental gene expression, which subsequently may lead to a risk of adverse perinatal outcomes [43-45]. However, after comparing our data regarding the altered transcriptome in the firsttrimester placenta with data from previous studies on the term placenta and endometrium during early implantation, we found that the IVF-ET procedure has a broader and more severe effect on early placentation $[21,22,46]$. Three term placentas from IVF-ET were examined using the same gene chip in our analysis, and only 18 differentially expressed genes and 6 biological functions were reported [21]. Another current study used 10 term placentas from IVF-ET for microarray analysis and identified 1866 differentially expressed genes, including 839 upregulated and 927 downregulated genes [22]. In addition, considering that modification of the maternal endometrium may affect trophoblast invasion and tissue remodeling, 641 differentially expressed genes were identified by microarray analysis from 23 samples from human endometrial biopsies [46]. Our results suggest that early placental tissue may undergo more extensive genetic and functional changes than term placental and other tissues from IVF-ET.

IPA of our data revealed that more than 50 pathways were disrupted in the first-trimester placenta derived from IVF-ET. Further analysis showed that these pathways are widely involved in key events of early placental development and function, whose disruption can lead to adverse outcomes [47-49]. We report the enrichment of multiple genes in the top 50 significantly overrepresented biological pathways. As expected, the first group of pathways comprise metabolic pathways. The IVF-ET process changes the physiological hypoxic environment of the trophoblast in vivo [50]. Metabolic disturbances may lead to metabolic reprogramming of the trophoblast in the first-trimester placenta [51]. Furthermore, the abnormal metabolic characteristics appear to be irreversible, even under normal conditions [52]. Abnormal metabolites, including prostaglandins, leukotrienes, lipid peroxidation and inflammatory mediation, may contribute to vasoconstriction and inflammatory syndrome, eventually resulting in adverse perinatal outcomes [53- 

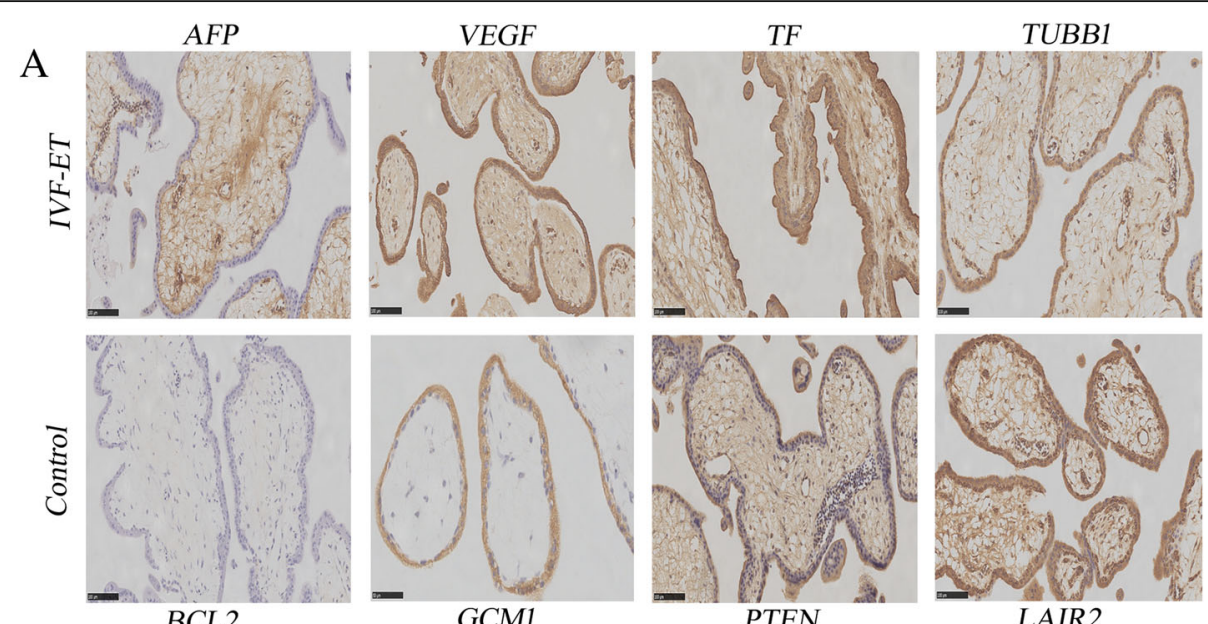

B
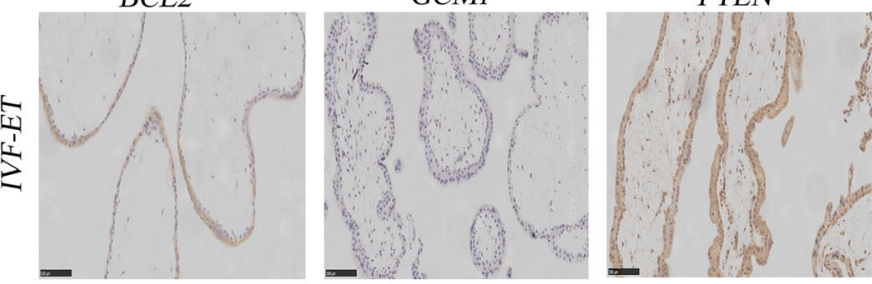

LAIR2
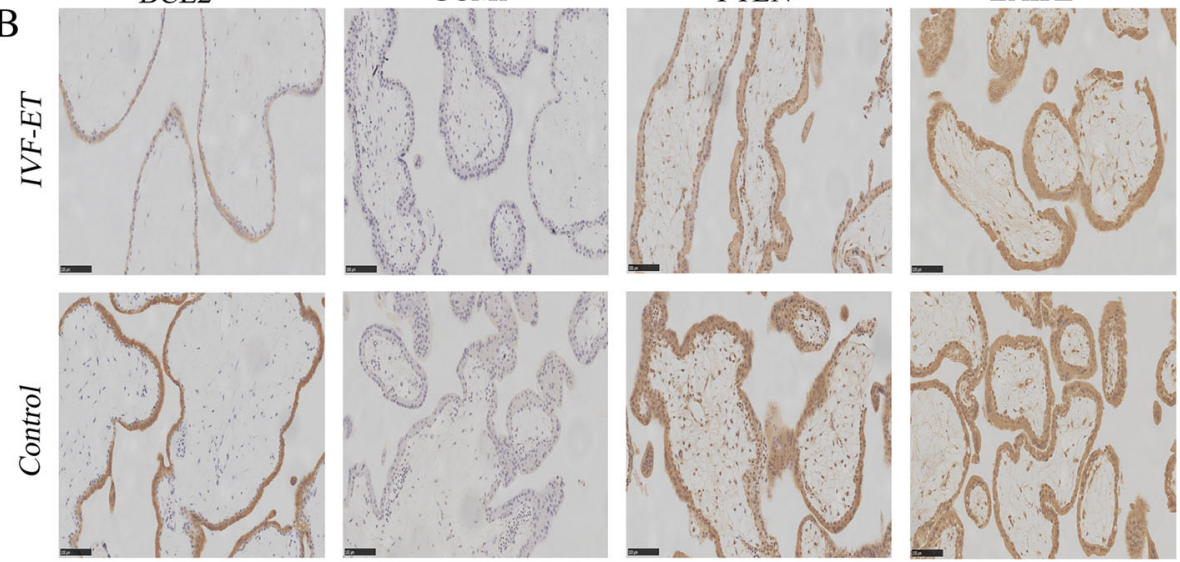

$\mathrm{C}$

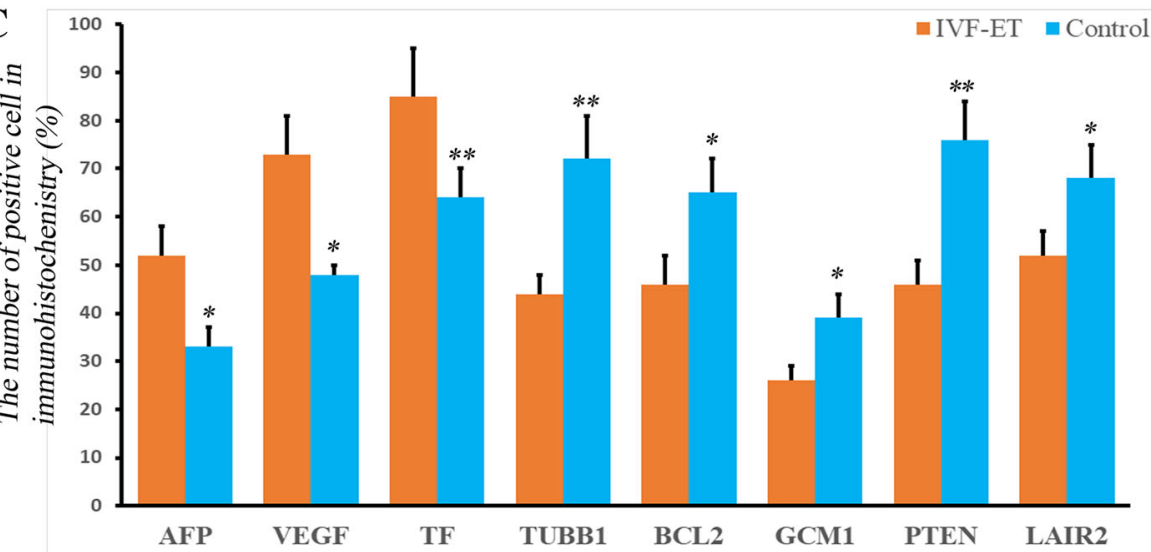

Fig. 5 Immunohistochemical analysis of the expression of 8 genes in the first-trimester placental tissues of the IVF-ET and control groups. a Immunohistochemical analysis of the expression levels of AFP, VEGF, TF and TUBB1 in the first-trimester placentas of the IVF-ET and control groups. $\mathbf{b}$ Immunohistochemical analysis of the expression levels of BCL2, GCM1, PTEN and LAIR2 in the first-trimester placentas of the IVF-ET and control groups. c Immunohistochemical analysis of the number of positively stained cells expressing the 8 genes in the IVF-ET and control groups. All data are expressed as the mean \pm standard deviation, * indicates $P<0.05$; ** indicates $P<0.01$, Scale bar $=100 \mu \mathrm{m}$

55]. Complement and coagulation cascades contribute to homeostasis, thrombosis, defense infection, immune tolerance and apoptotic cell clearance [56, 57]. Abnormalities in these cascades may lead to recurrent pregnancy loss, vascular complications and preeclampsia [58, 59].
The PPAR signaling pathway plays an important role in trophoblast fusion, migration, angiogenesis, lipid metabolism and trophoblast cycle progression [60]. Abnormalities in the PPAR signaling pathway may contribute to increased apoptosis of trophoblasts, trophoblast fusion 
disorder and necrosis of syncytiotrophoblasts, resulting in severe preeclampsia and intrauterine growth restriction. [61, 62]. However, in most cases involving humans, no immediate abortion has been observed, and most women give birth successfully after IVF-ET, which supports the hypothesis that interference by IVF-ET can trigger a placental compensatory mechanism [63, 64].

Moreover, regarding the compensatory mechanism in the first-trimester placenta after IVF-ET, we analyzed altered gene expression as a whole, rather than at the individual gene level. Interestingly, we separately classified upregulated genes and downregulated genes from IVFET sources according to their biological functions and found that the biological functions affected by these genes were consistent between the up- and downregulated genes. In each of the affected biological function categories, the total numbers of upregulated and downregulated genes are similar. From the perspective of signaling pathways, we simultaneously demonstrated and provided the locations of the up- and downregulated genes. Our labeled pathway maps demonstrate that multiple local functional groups are simultaneously affected by the upregulation and downregulation of these genes, resulting in a nearly balanced biological function. The compensatory mechanism in placental development is also observed in animal models. In early pregnancy, the placenta of the IVF-ET mouse pregnancy is smaller than that of the naturally conceived pregnancy [65], and in the third trimester and delivery stage, the placenta is heavier than that of the control [66]. Likewise, even after adjusting for confounding factors in IVF-ET, the human placenta and neonatal weight ratio are often higher than those of the natural control [67]. However, a heavier placenta does not represent more efficient transport of oxygen and nutrients [68]. Another study demonstrated that the weight and size of the placenta is associated with coronary disease and life expectancy [69, 70]. Nevertheless, the human placental compensatory mechanism can only reach a certain point. More severe disruptions can lead to immediate and subsequent adverse outcomes.

To our knowledge, our research is the first to demonstrate alterations in human first-trimester placental gene expression in vivo during early implantation. However, we understand that there are a few limitations with the design of our study. The first limitation is the sample size, but the results were strengthened by the fact that the samples were similar in terms of age, BMI and gestational weeks. The second limitation is twin pregnancy. Although we obtained first-trimester placental tissues in vivo using a fetal reduction strategy, a twin pregnancy has a potential impact on placentation and does not fully reflect a singleton pregnancy. In this study, we focused only on the effect of IVF-ET on the first-trimester placental function and compensatory mechanism in vivo.
Additionally, even though the samples were carefully examined under a microscope and stained by immunohistochemistry, we were still concerned about interference from maternal and embryonic cells. Nevertheless, we still believe that these altered gene expression patterns in the first-trimester placenta may partially explain the clinical characteristics and adverse perinatal outcomes of IVF-ET.

\section{Conclusion}

Our study describes the altered gene expression patterns in the early placenta of IVF-ET pregnancies compared to those in spontaneously conceived pregnancies. The abnormal biological functions and pathways associated with these differentially expressed genes provide a potential mechanism for understanding adverse perinatal outcomes following IVF-ET. Our contribution is the verification of a novel hypothesis that the increased risk of adverse perinatal outcomes in IVF-ET originates from early abnormal placentation. The initially disrupted placentation triggers a compensatory mechanism, and fortunately, most of these compensatory actions are successful. The innovation of our study is the examination of the first-trimester human placenta in vivo during the critical periods of implantation in IVF-ET, enabling relevant research to further investigate the target genes and pathways in vivo and not just in animal models. These data provide a potential basis for further analysis of the higher frequency of adverse perinatal outcomes following IVF-ET, with the ultimate goal of developing safer IVF-ET protocols.

\section{Additional files}

Additional file 1: Table S1. Detail information on the selection of primers for real-time RT-PCR experiments (DOC $34 \mathrm{~kb}$ )

Additional file 2: Table S2. The biological processes of up regulated genes in placental subjected to IVF-ET (DOC 68 kb)

Additional file 3: Table S3. The biological processes of down regulated genes in placental subjected to IVF-ET (DOC $70 \mathrm{~kb}$ )

Additional file 4: Figure S1. KEGG analysis of the differentially expressed genes, which are labeled, in the PPAR signaling pathway in the placenta after IVF-ET during the first trimester by KEGG. Red, upregulated; Blue, downregulated; Green, no change. (TIF $14811 \mathrm{~kb}$ )

\section{Abbreviations}

AFP: Alpha-fetoprotein; BCL2: B-cell CLL/lymphoma 2; BMI: Body mass index; DAVID: Database for annotation, visualization and integrated discovery; EGFR: Epidermal growth factor receptor; FDR: False discovery rate; GCM1: Glial cells missing homolog 1 (Drosophila);

IHC: Immunohistochemistry; IPA: Ingenuity pathway analysis; IVF-ET: In vitro fertilization and embryo transfer; KEGG: Kyoto encyclopedia of genes and genomes; KOBAS: KEGG orthology-based annotation system; LAIR2: Leukocyte-associated immunoglobulin-like receptor 2; MT1M: Metallothionein $1 \mathrm{M}$; PTEN: Phosphatase and tension homolog; RTqPCR: Real-time quantitative PCR; TF: Transferrin; TUBB1: Tubulin beta 1 class VI; VEGF: Vascular endothelial growth factor 


\section{Acknowledgments}

The authors appreciate the help from all patients enrolled in the Department of Gynecology and Obstetrics, Beijing Jishuitan Hospital, Beijing, China. We gratefully acknowledge the CapitalBio Corporation for conducting the RNA extractions and microarrays. The authors are also grateful to the anonymous reviewers for their constructive comments on the manuscript.

\section{Authors' contributions}

Dr. LFS designed the project and conducted the experimental study. LZ collected the samples, performed the experiments and wrote the manuscript. XLZ participated in designing the experiments and in writing and reviewing the manuscript. JFL was involved in performing some experiments. RZ were involved in the data collection and statistical analysis. $\mathrm{RY}$ and $\mathrm{YW}$ were involved in reviewing the manuscript. All authors read and approved the final manuscript.

\section{Funding}

The study was funded by the National Natural Science Foundation of China (No. 81070493). The funding bodies had no role in the study design, data collection and analysis, decision to publish, or preparation of the manuscript.

\section{Availability of data and materials}

The primary microarray data of the placental samples have been submitted to the Gene Expression Omnibus of the National Center for Biotechnology Information. The data can be obtained through GEO Series accession number GSE 122214 (https://www.ncbi.n/m.nih.gov/geo/query/acc.cgi?acc= GSE122214). Other data generated or analyzed during this study are available from the corresponding author upon reasonable request.

\section{Ethics approval and consent to participate}

The study was approved by the Ethics Committee of Beijing Jishuitan Hospital (Permission no 201703-11), and written informed consent was obtained from each individual prior to recruitment.

\section{Consent for publication}

Not applicable.

\section{Competing interests}

The authors declare that they have no competing interests.

\section{Author details}

'Department of Obstetrics and Gynecology, Beijing Jishuitan, Hospital, No. 31, Xinjiekou East Street, Xicheng District, Beijing 100035, People's Republic of China. ${ }^{2}$ Reproductive Medical Center, Department of Obstetrics and Gynecology, Peking University Third Hospital, No. 49, Huayuan North Road, Haidian District, Beijing 100191, People's Republic of China.

\section{Received: 26 March 2019 Accepted: 17 June 2019}

\section{Published online: 01 July 2019}

\section{References}

1. De Geyter C, Calhaz-Jorge C, Kupka MS, Wyns C, Mocanu E, Motrenko T, et al. ART in Europe, 2014: results generated from European registries by ESHRE: the European IVF-monitoring consortium (EIM) for the European Society of Human Reproduction and Embryology (ESHRE). Hum Reprod. 2018;33(9):1586-601.

2. Ishihara O, Araki R, Kuwahara A, Itakura A, Saito H, Adamson GD. Impact of frozen-thawed single-blastocyst transfer on maternal and neonatal outcome: an analysis of 277,042 single-embryo transfer cycles from 2008 to 2010 in Japan. Fertil Steril. 2014;101(1):128-33.

3. Sunderam S, Boulet SL, Jamieson DJ, Kissin DM. Effects of patient education on desire for twins and use of elective single embryo transfer procedures during ART treatment: a systematic review. Reprod Biomed Soc Online. 2018;6:102-19.

4. Wu Y, Chen W, Zhou L, Gao X, Xi X. Single embryo transfer improve the perinatal outcome in singleton pregnancy. J Matern Fetal Neonatal Med. 2019;27:1-6.

5. Henningsen AK, Pinborg A. Birth and perinatal outcomes and complications for babies conceived following ART. Semin Fetal Neonatal Med. 2014;19(4): 234-8.
6. Heo JS, Lee HJ, Lee MH, Choi CW. Comparison of neonatal outcomes of very low birth weight infants by mode of conception: in vitro fertilization versus natural pregnancy. Fertil Steril. 2019;111(5):962-70.

7. Yu B, Vega M, Zaghi S, Fritz R, Jindal S, Buyuk E. Comparison of perinatal outcomes following frozen embryo transfer cycles using autologous versus donor oocytes in women 40 to 43 years old: analysis of SART CORS data. J Assist Reprod Genet. 2018;35(11):2025-9.

8. Luke B, Gopal D, Cabral H, Diop H, Stern JE. Perinatal outcomes of singleton siblings: the effects of changing maternal fertility status. J Assist Reprod Genet. 2016;33(9):1203-13.

9. Declercq E, Luke B, Belanoff C, Cabral H, Diop H, Gopal D, et al. Perinatal outcomes associated with assisted reproductive technology: the Massachusetts outcomes study of assisted reproductive technologies (MOSART). Fertil Steril. 2015;103(4):888-95.

10. Zhu L, Zhang Y, Liu Y, Zhang R, Wu Y, Huang Y, et al. Maternal and livebirth outcomes of pregnancies following assisted reproductive technology: a retrospective cohort study. Sci Rep. 2016;6:35141.

11. Toschi P, Czernik M, Zacchini F, Fidanza A, Loi P, Ptak GE. Evidence of placental autophagy during early pregnancy after transfer of in vitro produced (IVP) sheep embryos. PLoS One. 2016;11(6):e0157594.

12. Rivera RM, Stein P, Weaver JR, Mager J, Schultz RM, Bartolomei MS. Manipulations of mouse embryos prior to implantation result in aberrant expression of imprinted genes on day 9.5 of development. Hum Mol Genet. 2008;17(1):1-14.

13. Fauque $\mathrm{P}$, Mondon $\mathrm{F}$, Letourneur $\mathrm{F}$, Ripoche MA, Journot $\mathrm{L}$, Barbaux $\mathrm{S}$, et al. In vitro fertilization and embryo culture strongly impact the placental transcriptome in the mouse model. PLoS One. 2010;5(2):e9218.

14. Kurowski A, Molotkov A, Soriano P. FGFR1 regulates trophectoderm development and facilitates blastocyst implantation. Dev Biol. 2019;446(1): 94-101.

15. Mann MR, Lee SS, Doherty AS, Verona RI, Nolen LD, Schultz RM, et al. Selective loss of imprinting in the placenta following preimplantation development in culture. Development. 2004;131(15):3727-35.

16. Yao J, Geng L, Huang R, Peng W, Chen X, Jiang X, et al. Effect of vitrification on in vitro development and imprinted gene Grb10 in mouse embryos. Reproduction. 2017;154(3):97-105.

17. Zandstra H, Brentjens LBPM, Spauwen B, Touwslager RNH, Bons JAP, Mulder $A L$, et al. Association of culture medium with growth, weight and cardiovascular development of IVF children at the age of 9 years. Hum Reprod. 2018;33(9):1645-56.

18. Fauque P, Ripoche MA, Tost J, Journot L, Gabory A, Busato F, et al. Modulation of imprinted gene network in placenta results in normal development of in vitro manipulated mouse embryos. Hum Mol Genet. 2010;19(9):1779-90.

19. Ghosh J, Mainigi M, Coutifaris C, Sapienza C. Outlier DNA methylation levels as an indicator of environmental exposure and risk of undesirable birth outcome. Hum Mol Genet. 2016;25(1):123-9.

20. Ghosh J, Coutifaris C, Sapienza C, Mainigi M. Global DNA methylation levels are altered by modifiable clinical manipulations in assisted reproductive technologies. Clin Epigenetics. 2017;9:14.

21. Zhang Y, Cui Y, Zhou Z, Sha J, Li Y, Liu J. Altered global gene expressions of human placentae subjected to assisted reproductive technology treatments. Placenta. 2010:31(4):251-8.

22. Nelissen EC, Dumoulin JC, Busato F, Ponger L, Eijssen LM, Evers JL, et al. Altered gene expression in human placentas after IVF/ICSI. Hum Reprod. 2014;29(12):2821-31.

23. Duranthon V, Chavatte-Palmer P. Long term effects of ART: what do animals tell us? Mol Reprod Dev. 2018;85(4):348-68.

24. Hossain MM, Tesfaye D, Salilew-Wondim D, Held E, Pröll MJ, Rings F, et al. Massive deregulation of miRNAs from nuclear reprogramming errors during trophoblast differentiation for placentogenesis in cloned pregnancy. BMC Genomics. 2014;15:43.

25. de Waal E, Mak W, Calhoun S, Stein P, Ord T, Krapp C, et al. In vitro culture increases the frequency of stochastic epigenetic errors at imprinted genes in placental tissues from mouse concepti produced through assisted reproductive technologies. Biol Reprod. 2014;90(2):22.

26. Fisher SJ. Why is placentation abnormal in preeclampsia? Am J Obstet Gynecol. 2015;213(4 Suppl):S115-22.

27. Louwen F, Muschol-Steinmetz C, Reinhard J, Reitter A, Yuan J. A lesson for cancer research: placental microarray gene analysis in preeclampsia. Oncotarget. 2012;3(8):759-73. 
28. Zou K, Ding G, Huang H. Advances in research into gamete and embryofetal origins of adult diseases. Sci China Life Sci. 2019;62(3):360-8.

29. Leijnse JEW, de Heus R, de Jager W, Rodenburg W, Peeters LLH, Franx A, et al. First trimester placental vascularization and angiogenetic factors are associated with adverse pregnancy outcome. Pregnancy Hypertens. 2018;13:87-94.

30. Yang R, Liu YY, Zhao L, Wang Y, Li R, Liu P, et al. Human trophoblast cell during first trimester after IVF-ET differs from natural conceived pregnancy in development and function. Histol Histopathol. 2017;32(3):243-51.

31. Yue $X L$, Gao ZQ. Identification of pathogenic genes of pterygium based on the gene expression omnibus database. Int J Ophthalmol. 2019;12(4):529-35.

32. Kapourani CA, Sanguinetti G. BPRMeth: a flexible Bioconductor package for modelling methylation profiles. Bioinformatics. 2018;34(14):2485-6.

33. Maheshwari A, Pandey S, Shetty A, Hamilton M, Bhattacharya S. Obstetric and perinatal outcomes in singleton pregnancies resulting from the transfer of frozen thawed versus fresh embryos generated through in vitro fertilization treatment: a systematic review and meta-analysis. Fertil Steril. 2012;98(2):368-77.

34. Imudia AN, Awonuga AO, Kaimal AJ, Wright DL, Styer AK, Toth TL. Elective cryopreservation of all embryos with subsequent cryothaw embryo transfer in patients at risk for ovarian hyperstimulation syndrome reduces the risk of adverse obstetric outcomes: a preliminary study. Fertil Steril. 2013;99(1):168-73.

35. Sharpe RM. Of mice and men: long-term safety of assisted reproduction treatments. Hum Reprod. 2018;33(5):793-796.

36. Donjacour A, Liu X, Lin W, Simbulan R, Rinaudo PF. In vitro fertilization affects growth and glucose metabolism in a sex-specific manner in an outbred mouse model. Biol Reprod. 2014;90(4):80.

37. Pinborg A, Wennerholm UB, Romundstad LB, Loft A, Aittomaki K, Söderström-Anttila $V$, et al. Why do singletons conceived after assisted reproduction technology have adverse perinatal outcome? Systematic review and meta-analysis. Hum Reprod Update. 2013;19(4):87-104.

38. Turco MY, Gardner L, Kay RG, Hamilton RS, Prater M, Hollinshead MS, et al. Trophoblast organoids as a model for maternal-fetal interactions during human placentation. Nature. 2018;564(7735):263-7.

39. Feng $Y, M a X$, Deng $L, Y a o B$, Xiong $Y$, Wu $Y$. Role of selectins and their ligands in human implantation stage. Glycobiology. 2017;27(5):385-91.

40. Evans J. Hyperglycosylated hCG: a unique human implantation and invasion factor. Am J Reprod Immunol. 2016;75(3):333-40.

41. James JL, Carter AM, Chamley LW. Human placentation from nidation to 5 weeks of gestation. Part II: tools to model the crucial first days. Placenta. 2012;33(5):335-42.

42. Xie L, Sadovsky Y. The function of miR-519d in cell migration, invasion, and proliferation suggests a role in early placentation. Placenta. 2016;48:34-7.

43. Litzky JF, Deyssenroth MA, Everson TM, Armstrong DA, Lambertini L, Chen J, et al. Placental imprinting variation associated with assisted reproductive technologies and subfertility. Epigenetics. 2017;12(8):653-61.

44. Xu N, Barlow GM, Cui J, Wang ET, Lee B, Akhlaghpour M, et al. Comparison of genome-wide and gene-specific DNA methylation profiling in firsttrimester chorionic villi from pregnancies conceived with infertility treatments. Reprod Sci. 2017;24(7):996-1004

45. Thomopoulos C, Tsioufis C, Michalopoulou H, Makris T, Papademetriou V, Stefanadis C. Assisted reproductive technology and pregnancy-related hypertensive complications: a systematic review. J Hum Hypertens. 2013;27(3):148-57.

46. Senapati S, Wang F, Ord T, Coutifaris C, Feng R, Mainigi M. Superovulation alters the expression of endometrial genes critical to tissue remodeling and placentation. J Assist Reprod Genet. 2018;35(10):1799-808.

47. Nevalainen J, Skarp S, Savolainen ER, Ryynänen M, Järvenpää J. Intrauterine growth restriction and placental gene expression in severe preeclampsia, comparing early-onset and late-onset forms. J Perinat Med. 2017:45(7):869-77.

48. Sibley CP. Treating the dysfunctional placenta. J Endocrinol. 2017;234(2):R81-97.

49. Gao F, Hu W, Li Y, Shen H, Hu J. Mono-2-ethylhexyl phthalate inhibits human extravillous trophoblast invasion via the PPARY pathway. Toxicol Appl Pharmacol. 2017:327:23-9.

50. Puscheck EE, Bolnick A, Awonuga A, Yang Y, Abdulhasan M, Li Q, et al. Why AMPK agonists not known to be stressors may surprisingly contribute to miscarriage or hinder IVF/ART. J Assist Reprod Genet. 2018;35(8):1359-66.

51. Knyazev EN, Zakharova GS, Astakhova LA, Tsypina IM, Tonevitsky AG, Sukhikh GT. Metabolic reprogramming of trophoblast cells in response to hypoxia. Bull Exp Biol Med. 2019;166(3):321-5.

52. Dunn WB, Brown M, Worton SA, Crocker IP, Broadhurst D, Horgan R, et al. Changes in the metabolic footprint of placental explant-conditioned culture medium identifies metabolic disturbances related to hypoxia and preeclampsia. Placenta. 2009;30(11):974-80.
53. Mitsuya K, Parker AN, Liu L, Ruan J, Vissers MCM, Myatt L. Alterations in the placental methylome with maternal obesity and evidence for metabolic regulation. PLoS One. 2017;12(10):e0186115.

54. Murthi P, Wallace EM, Walker DW. Altered placental tryptophan metabolic pathway in human fetal growth restriction. Placenta. 2017;52:62-70.

55. Kiang JG, Krishnan S, Lu X, Li Y. Inhibition of inducible nitric-oxide synthase protects human T cells from hypoxia-induced apoptosis. Mol Pharmacol. 2008;73(3):738-47.

56. Jin X, Ma Q, Sun Z, Yang X, Zhou Q, Qu G, et al. Airborne fine particles induce hematological effects through regulating the crosstalk of the Kallikrein-Kinin, complement, and coagulation systems. Environ Sci Technol. 2019;53(3):2840-51.

57. Levi M, Cohn DM, Zeerleder S. Hereditary angioedema: linking complement regulation to the coagulation system. Res Pract Thromb Haemost. 2019;3(1):38-43.

58. Than NG, Romero R, Tarca AL, Kekesi KA, Xu Y, Xu Z, et al. Integrated systems biology approach identifies novel maternal and placental pathways of preeclampsia. Front Immunol. 2018;9:1661.

59. Scully M, Cataland S, Coppo P, de la Rubia J, Friedman KD, Kremer Hovinga $\mathrm{J}$, et al. Consensus on the standardization of terminology in thrombotic thrombocytopenic purpura and related thrombotic microangiopathies. J Thromb Haemost. 2017;15(2):312-22

60. Ruebner M, Langbein M, Strissel PL, Henke C, Schmidt D, Goecke TW, et al. Regulation of the human endogenous retroviral Syncytin-1 and cell-cell fusion by the nuclear hormone receptors PPARY/RXRa in placentogenesis. J Cell Biochem. 2012;113(7):2383-96.

61. Ganss R. Maternal metabolism and vascular adaptation in pregnancy: the PPAR link. Trends Endocrinol Metab. 2017;28(1):73-84.

62. Allam HIG, Masri AAA. The potential therapeutic role of peroxisome ProliferatorActivated receptors agonist in Preeclamptic pregnant rats. J Coll Physicians Surg Pak. 2018;28(1):31-5.

63. Higgins JS, Vaughan OR, Fernandez de Liger E, Fowden AL, Sferruzzi-Perri AN. Placental phenotype and resource allocation to fetal growth are modified by the timing and degree of hypoxia during mouse pregnancy. J Physiol. 2016;594(5):1341-56.

64. Kouhkan A, Khamseh ME, Pirjani R, Moini A, Arabipoor A, Maroufizadeh S, et al. Obstetric and perinatal outcomes of singleton pregnancies conceived via assisted reproductive technology complicated by gestational diabetes mellitus: a prospective cohort study. BMC Pregnancy Childbirth. 2018;18(1):495.

65. Delle Piane L, Lin W, Liu X, Donjacour A, Minasi P, Revelli A, et al. Effect of the method of conception and embryo transfer procedure on midgestation placenta and fetal development in an IVF mouse model. Hum Reprod. 2010;25(8):2039-46.

66. Bloise E, Lin W, Liu X, Simbulan R, Kolahi KS, Petraglia F, et al. Impaired placental nutrient transport in mice generated by in vitro fertilization. Endocrinology. 2012;153(7):3457-67.

67. Haavaldsen C, Tanbo T, Eskild A. Placental weight in singleton pregnancies with and without assisted reproductive technology: a population study of 536,567 pregnancies. Hum Reprod. 2012;27(2):576-82.

68. Mirbod P. Analytical model of the feto-placental vascular system: consideration of placental oxygen transport. R Soc Open Sci. 2018;5(4):180219.

69. Dahdouh S, Andescavage N, Yewale S, Yarish A, Lanham D, Bulas D, et al. In vivo placental MRI shape and textural features predict fetal growth restriction and postnatal outcome. J Magn Reson Imaging. 2018;47(2):449-58.

70. Barker DJ, Thornburg KL. Placental programming of chronic diseases, cancer and lifespan: a review. Placenta. 2013;34(10):841-5.

\section{Publisher's note}

Springer Nature remains neutral with regard to jurisdictional claims in published maps and institutional affiliations. 